Sachverhalt hat das OLG - erneut im Rahmen der gebotenen tatrichterlichen Würdigung - verneint und insoweit ausgeführt, die besondere Behandlung in einem NeonatologieZentrum von Anfang an wäre zwar wünschenswert gewesen, Ansatzpunkte bei Übernahme der Behandlung, welche eine Einweisung oder Behandlung in einem NeonatologieZentrum zwingend gefordert hätten, seien jedoch nicht gegeben gewesen; es hätten nach der Geburt zunächst keine Anhaltspunkte für eine Notfallsituation bestanden.

5. Der $\mathrm{BGH}$ ist derzeit mit der Angelegenheit befasst (Az.: VI ZA 1/21).

https://doi.org/10.1007/s00350-021-6014-8

\section{Werbung für eine Fernbehandlung nach Novellierung des §9 HWG}

\section{HWG §9, UWG §3a}

1. Nach $₫ 9$ S. 2 HWG ist S. 1 - an dem sich durch die Neufassung der Vorschrift nichts geändert hat - nicht anzuwenden auf die Werbung für Fernbehandlungen, die unter Verwendung von Kommunikationsmedien erfolgen, wenn nach allgemein anerkannten fachlichen Standards ein persönlicher ärztlicher Kontakt mit dem zu behandelnden Menschen nicht erforderlich ist. $\$ 9$ S. 2 HWG wurde in die Vorschrift aufgenommen, um die Anpassung des ärztlichen Berufsrechtes im Hinblick auf die Reichweite des Werbeverbotes nachzuvollziehen.

2. Maßstab für die ,allgemein anerkannten fachlichen Standards" gemäß $₫ 9$ S. 2 HWG ist die MBOAnders als bei der Beurteilung eines konkreten Arztverhaltens im Einzelfall, kommt es für die abstrakte, generalisierende Bewertung des $\$ 9 \mathrm{HWG}$ nicht auf die einzelnen Vorschriften der verschiedenen Landesberufsordnungen an.

3. Das streitgegenständliche Setting berücksichtigt nicht die notwendige Einzelfallprüfung des $₫ 7$ Abs. 4 MBO- $\ddot{A}$ und entspricht insoweit nicht den ,allgemein anerkannten fachlichen Standards" i.S.d. $\$ 9$ S. 2 HWG. Der „Einzelfall“" i.S.d. $₫ 7$ Abs. 4 S. 3 MBO$\ddot{A}$ meint das konkrete Arzt-Patienten-Verhältnis und nicht ein spezielles Krankheitsbild, wie z.B. das einer Erkältung. (Leitsätze der Bearbeiterin)

OLG Hamburg, Urt. v. 5. 11.2020 - 5 U 175/19 (LG Hamburg)

Problemstellung: Die Entscheidung des OLG Hamburg befasst sich mit der im Rahmen der zunehmenden Digitalisierung im Gesundheitswesen höchst aktuellen Frage der Werbung für eine Fernbehandlung. Gegenstand der Beurteilung ist die wettbewerbsrechtliche Zulässigkeit eines Geschäftsmodells, welches bei Erkältungserscheinungen eine Krankschreibung per WhatsApp allein auf der Grundlage von durch den $\mathrm{Pa}-$ tienten auf der Homepage des Anbieters online ausgefüllten Fragebögen ermöglicht, ohne eine Verifizierung der Angaben per Telefon oder Videosprechstunde mit einem Telemediziner vorzusehen.

Es handelt sich hierbei nach dem Urt. des OLG München (MedR 2021, 154 m. Anm. Braun) zu der Zulässigkeit der Werbung für einen digitalen Arztbesuch soweit ersichtlich - erst um die zweite obergerichtliche Entscheidung, die sich mit dem Ausnahmetatbestand des novellierten \$9 HWG befasst. \$9 HWG n. F. wurde auf-

Eingesandt von Rechtsanwalt Prof. Dr. iur. Franz-Josef Dahm, Essen; bearbeitet von Rechtsanwältin Dr. iur. Corinna Schmidt-Murra, SCHMIDT, VON DER OSTEN \& HUBER

Rechtsanwälte Steuerberater Partnerschaft mbB,

Rüttenscheider Str. 26, 45128 Essen, Deutschland grund einer deutlichen Liberalisierung der eine Fernbehandlung betreffenden standesrechtlichen Vorschriften der Ärzte zum 19.12.2019 um einen S. 2 ergänzt, der das nunmehr als S. 1 gefasste, vormals geltende generelle Verbot einer Werbung für eine Fernbehandlung um einen Erlaubnisvorbehalt ergänzt. Demnach ist S. 1 nicht anzuwenden auf die Werbung für Fernbehandlungen, die unter Verwendung von Kommunikationsmedien erfolgen, wenn nach allgemein anerkannten fachlichen Standards ein persönlicher ärztlicher Kontakt mit dem $\mathrm{zu}$ behandelnden Menschen nicht erforderlich ist.

Das Gericht nimmt nunmehr erstmals eine Konkretisierung des Merkmals der allgemein anerkannten fachlichen Standards vor, welche durch das Gesetz nicht unmittelbar selbst definiert werden. Bei seiner diesbezüglichen Bezugnahme auf die MBO- $\mathrm{A}$ muss es sich hierbei auch mit der Relevanz eines abweichenden Wortlauts in der einschlägigen $\mathrm{BO}$ der Landesärztekammer Schleswig-Holstein befassen. Denn anders als $\$ 7 \mathrm{Abs} .4$ S. $3 \mathrm{MBO}-\ddot{A}$ enthält $\$ 7$ Abs. 4 S. 3 BO-Ä SchleswigHolstein gerade nicht das Merkmal, dass nur ,im Einzelfall" eine Beratung oder Behandlung ausschließlich über Kommunikationsmedien erlaubt ist, wenn diese ärztlich vertretbar und ein persönlicher Kontakt mit der Patientin oder dem Patienten nicht erforderlich ist. In diesem Zusammenhang stellt sich sodann auch die Frage, welche Bedeutung das individuelle Arzt-PatientenVerhältnis für die Zulässigkeit einer abstrakt-generellen Werbung für eine Fernbehandlung erlangen kann.

Anders als die erste Instanz (MedR 2020, 391 m. Anm. Hahn, MedR 2020, 370), die sich allein mit der (Un-)Zulässigkeit der Ausstellung ärztlicher Atteste bei einer Fernbehandlung am Maßstab des $₫ 25 \mathrm{MBO}-\ddot{\mathrm{A}}$ befasste, unterlässt das OLG Hamburg bedauerlicherweise eine Auseinandersetzung mit der Frage, ob für die Ausstellung eines Attestes bei einer ausschließlichen Fernbehandlung andere bzw. zusätzliche Zulässigkeitsmaßstäbe berücksichtigt werden müssen.

Corinna Schmidt-Murra

Zum Sachverhalt: Der Kl. verfolgt lauterkeitsrechtliche Unterlassungsansprüche. Daneben begehrt er die Bezahlung von vorgerichtlich zum Zwecke der Abmahnung entstandenen Aufwendungen.

Der Kl. ist ein eingetragener Verein $(A G \ldots-N z \ldots)$, zu dessen satzungsmäßigen Aufgaben die Wahrung der gewerblichen Interessen seiner Mitglieder, insbesondere die Achtung darauf gehört, dass die Regeln des lauteren Wettbewerbs eingehalten werden. Zu den Mitgliedern des K1. gehören unter anderem die Ärztekammern H. und S., die Zahnärztekammer S., der Bundesverband der ... e. V., 13 Ärzte und vier Kliniken. Der Kl. ist nach seiner personellen, sachlichen und finanziellen Ausstattung in der Lage, seine satzungsgemäBen Aufgaben wahrzunehmen.

Die Bekl. ermöglicht den Erhalt einer Arbeitsunfähigkeitsbescheinigung (im Folgenden: AU-Bescheinigung) ausschließlich im Wege der Fernbehandlung. Der Interessierte kann hierzu über sein Mobiltelefon die Internetseite au... .de aufrufen. Unter dem Link „Los geht's“ erhält er folgendes Angebot (Anlage K 5):

,Sie sind arbeitsunfähig wegen Erkältung und müssten daher zum Arzt? Hier erhalten Sie Ihre AU-Bescheinigung einfach online per Handy nach Hause!

Wenn Sie werktags (Mo-Fr) vor 10 Uhr bestellen, versenden wir Ihre AU bis 15 Uhr per WhatsApp \& per Post. Anderenfalls am nächsten Werktag (Mo-Fr) bis 15 Uhr. Beginn der AU ist immer das Bestelldatum."

Wird der Link angewählt, sind online verschiedene Fragen zu beantworten, die vor allem Symptome und mögliche Risikofaktoren betreffen. Führen die Antworten des Nutzers nicht zu einer Erkältungsdiagnose oder lassen sie die Zugehörigkeit zu einer Risikogruppe vermuten, wird der Nutzer mittels einer automatisch generierten Erklärung darauf hingewiesen, dass er den Dienst nicht nutzen kann. Im Anschluss kann der Dienst erneut in Anspruch genommen werden, ohne dass die zuvor gegebenen Antworten dabei berücksichtigt werden. Der Vorgang kann beliebige Male wiederholt werden. 
Wenn die mitgeteilten Symptome mit der Diagnose einer Erkältung übereinstimmen, werden die Antworten online an den ,TeleArzt" übermittelt, der die mitgeteilten Symptome überprüft und im Fall einer Erkältungsdiagnose die entsprechende AU-Bescheinigung ausstellt. Die AU-Bescheinigung wird per WhatsApp und per Post an den Nutzer geschickt. Für das Angebot verlangt die Bekl. einen Betrag i. H. v. 9,00€, der unabhängig vom Erfolgsfall zu entrichten ist und dem Nutzer von seiner Krankenkasse oder privaten Krankenversicherung nicht erstattet wird. Diesen Dienst bewirbt die Bekl. insbesondere mit den im Unterlassungsantrag wiedergegebenen Aussagen wie in Anlage K 5 festgehalten.

Der Kl. mahnte die Bekl. mit Schreiben v. 19. 2.2019 ab. Die Bek1. wies die Vorwürfe mit Schreiben vom selben Tag zurück.

Der Kl. hat vorgetragen, die Bekl. verstoße gegen $\$ 9 \mathrm{HWG}$, da sie für eine Fernbehandlung werbe, nämlich für eine Erkennung von Krankheiten, die nicht auf der unmittelbaren Wahrnehmung des Arztes beruhe. Darüber hinaus liege auch ein Verstoß gegen ärztliches Berufsrecht, nämlich $\$ \$ 7,11,25 \mathrm{MBO}-\mathrm{A}$, vor.

Im Rahmen der mündlichen Verhandlung vor dem LG Hamburg hat der Klägervertreter die angekündigten Klageanträge dahingehend klargestellt, dass die Werbung mit den zu Ziffer I. 1. bis 6 . beanstandeten Äußerungen nur in der konkreten Verletzungsform, wie in Anlage K 5 geschehen, verboten werden soll.

Der Kl. hat beantragt:

I. Die Bekl. wird verurteilt, es bei Meidung eines für jeden Fal der Zuwiderhandlung $\mathrm{zu}$ verhängenden Ordnungsgeldes bis $\mathrm{zu}$ $250.000,00 €$, ersatzweise Ordnungshaft, oder einer Ordnungshaft bis zu sechs Monaten, zu vollziehen am Geschäftsführer, zu unterlassen,

im geschäftlichen Verkehr Krankschreibungen/Arbeitsunfähigkeitsbescheinigungen zu bewerben,

wenn dies geschieht wie in Anlage K 5 wiedergegeben,

insbesondere wie in Anlage K 5 geschehen, mit den Angaben zu bewerben

1. Bei Erkältung erhalten Sie für $9,00 €$ eine gültige Krankschreibung vom Tele-Arzt über WhatsApp und per Post.

2. Sie sind arbeitsunfähig wegen Erkältung und müssten daher zum Arzt? Hier erhalten Sie Ihre AU-Bescheinigung einfach online per Handy nach Hause! Wenn Sie werktags (Mo-Fr) vor 10:00 Uhr bestellen, versenden wir Ihre AU bis 15:00 Uhr per WhatsApp und per Post. Anderenfalls am nächsten Werktag (Mo-

Fr) bis 15:00 Uhr. Beginn der AU ist immer das Bestelldatum.

3. Und so geht's: Symptome schicken, Risiken ausschließen, Daten eingeben, einfach bezahlen, fertig.

4. Das Besondere an einer Arzt-Behandlung per Telemedizin ist grundsätzlich, dass der Arzt keinen persönlichen Kontakt zu Ihnen hat. Daher kann er Sie insbesondere für eine Diagnose nicht körperlich untersuchen und ist insbesondere auf Anamnese angewiesen, d.h. Ihre Antworten auf seine Fragen insbesondere zu vorliegenden Symptomen.

5. Wir haben uns auf die Diagnose von Erkältungen konzentriert, da sie ungefährlich, gut erforscht und besonders gut per Anamnese zu diagnostizieren sind.

6. Unser Service für AU-Scheine per Handy bringt folgende Vorteile:

6.1. Sie verschwenden nicht Ihre wertvolle Genesungszeit für einen Arztbesuch.

6.2. Sie stecken niemanden im Wartezimmer an.

6.3. Sie können die Anfrage an einen Arzt rund um die Uhr absenden und sie wird zeitnah beantwortet.

6.4. Zur einfachen Kommunikation nutzen Sie die Ihnen vertraute und sichere App WhatsApp.

6.5. Sie erhalten vorformulierte Therapieempfehlungen in gleichbleibend hoher Qualität, die vorher mit ärztlicher Hilfe besonders sorgfältig formuliert wurden.

6.6. Ihre Behandlung ist auf Ihrem Handy dokumentiert.

6.7. Die Fehldiagnoserate ist geringer, da durch den Fragenkatalog Risikopatienten und solche Patienten aussortiert werden, welche Symptome aufweisen, die bei Erkältung statistisch nie vorkommen. Zudem sind die Ärzte durch ihre Tätigkeit für uns auf Erkältung spezialisiert und arbeiten mit Checklisten, welche die Fehldiagnoserate nachweislich verringern. Über WhatsApp erfragen wir zudem später bei Ihnen die Korrektheit der Diagnose, um so automatisiert aus etwaigen Fehlern zu lernen. $[\ldots]$

Die Bekl. hat vorgetragen, dass kein Verstoß gegen $₫ 9$ HWG vorliege. Es fehle der Produktbezug auf der Webseite, da es sich nur um eine sachliche Aufklärung über die Dienstleistung handele. Zudem sei nach dieser Vorschrift nach verfassungskonformer Auslegung nur Werbung für verbotene Telemedizin untersagt, was vorliegend nich der Fall sei. Ferner lägen auch keine Verstöße gegen die MBO-Ä vor. Alle seit Ende Januar 2019 für sie, die Bekl., tätigen Ärzte seien kein Mitglied der Ärztekammer H. gewesen. Der seit April 2019 für sie tätige Arzt sei Mitglied der Ärztekammer S., weshalb die Vorschriften der BOÄ-SH anwendbar seien. Danach sei eine Behandlung ausschließlich über Kommunikationsmedien nicht nur im Einzelfall, sondern generell erlaubt.

Die Klage ist der Bekl. am 24.4.2019 zugestellt worden. Das LG Hamburg hat die Bekl. durch Urt. v. 3.9.2019 antragsgemäß verurteilt. Wegen der Einzelheiten wird auf das genannte Urt. Bezug genommen. Mit der vorliegenden Berufung verfolgt die Bekl. ihr erstinstanzliches Begehren, die Klage abzuweisen, in vollem Umfang weiter.

Die Bekl. meint, das landgerichtliche Urt. sei in tatsächlicher sowie rechtlicher Hinsicht nicht haltbar. Sie vertieft insoweit ihren erstinstanzlichen Vortrag.

Des Weiteren trägt die Bekl. vor, das LG habe zu ihren Lasten das Recht auf rechtliches Gehör verletzt, indem es unberücksichtigt gelassen habe, dass ihr, der Bekl., bei bisher über 70.000 Ferndiagnosen keinerlei Fehldiagnosen gemeldet worden seien. Ferner sei mit dem Merkmal des „Einzelfalls“ in $\$ 7$ Abs. 4 S. 3 MBO-Ä nicht das konkrete Arzt-Patienten-Verhältnis, sondern vielmehr das spezielle Krankheitsbild gemeint. Da der für sie tätige Arzt seinen Sitz in S. habe, sei ausschließlich auf die Vorschriften der BOÄ-SH abzustellen. Diese setzten keine Prüfung im Einzelfall voraus.

Die Rechtsauffassung des LG führe dazu, dass telemedizinische Behandlungen faktisch niemals möglich wären, was dem offensichtlichen Willen des Gesetzgebers widerspreche. Schließlich habe der für die Berufsregeln der Kassenärzte zuständige G-BA Krankschreibungen bei leichten Atemwegserkrankungen ausdrücklich bis zu 14 Tage ohne persönlichen Kontakt erlaubt.

$[\ldots$

Der Kl. ist der Auffassung, es liege aus den bereits im erstinstanzlichen Verfahren vorgebrachten Gründen ein Verstoß gegen $\$ 9$ HWG vor. $\$ 9 \mathrm{HWG}$ sei ein abstraktes Gefährdungsdelikt, so dass es keine Rolle spiele, ob die Durchführung von Fernbehandlungen tatsächlich beabsichtigt oder erfolgt sei. Den auf $₫ 9 \mathrm{HWG}$ gestützten Anspruch habe das LG nicht zurückgewiesen, weshalb dieser weiterhin - ohne eingelegte Anschlussberufung - unter diesem Gesichtspunkt begründet werden könne. Ferner stehe $\$ 9 \mathrm{HWG}$ auch nicht im Widerspruch zu $₫ 7$ Abs. 4 MBO-Ä. Darüber hinaus lägen mehrere Verstöße gegen die ärztlichen Berufspflichten in Mittäterschaft der Bekl. vor.

$[\ldots]$

Aus den Gründen: II. Die zulässige Berufung der Bekl. ist unbegründet. Zu Recht hat das LG Hamburg der Klage vollumfänglich stattgegeben. Lediglich klarstellend ist im landgerichtlichen Ausspruch der eingefügte Zusatz ,wie in Anlage K 5 geschehen," nach dem Wort „,insbesondere“ zu streichen. Die auch nach dieser Streichung im Ausspruch weiterhin vorhandene Bezugnahme auf die konkrete Verletzungsform in Gestalt der Formulierung ,wenn dies geschieht wie in Anlage K 5 wiedergegeben", der dann zur weiteren Erläuterung die unter den Ziffern 1. bis 6. zitierten Angaben nachfolgen, konkretisiert das ausgesprochene Verbot hinreichend.

1. Dem Kl. steht der mit dem Klageantrag zu I. geltend gemachte Anspruch auf Unterlassung ebenso wie der mit dem Klageantrag zu II. verfolgte Anspruch auf Ersatz der Abmahnkosten zu.

a) Der Unterlassungsanspruch ergibt sich aus $\int \$ 8$ Abs. 1 S. 1, Abs. 3 Nr. 2, 3, 3a UWG i.V. mit $\$ 9$ HWG. Eine Anschlussberufung des K1. hinsichtlich des bereits erstinstanzlich gerügten, vom LG indes nicht behandelten Verstoßes gegen $\$ 9 \mathrm{HWG}$ ist nicht veranlasst gewesen. Es liegt ein einheitlicher Streitgegenstand vor. Insoweit ist auch kein neuer Tatsachenvortrag notwendig.

a) Der Kl. ist aktivlegitimiert gemäß $₫ 8$ Abs. 3 Nr. 2 UWG. Nach dem von der Bekl. nicht in Abrede genommenen Vortrag des Kl. ist dieser ein eingetragener Verein $(A G \ldots-\mathrm{Nz} \ldots$... , zu dessen satzungsmäßigen Aufgaben die Wahrung der gewerblichen Interessen seiner Mitglieder, insbesondere die Achtung darauf gehört, dass die Regeln 
des lauteren Wettbewerbs eingehalten werden. Dem K1. gehört eine erhebliche Anzahl Mitglieder an, die selbst bzw. deren Mitglieder „Waren oder Dienstleistungen“ gleicher oder verwandter Art wie diejenigen der Bekl. vertreiben, u. a. die Ärztekammern H. und S., die Zahnärztekammer S., 13 Ärzte und vier Kliniken. Der Kl. ist nach seiner personellen, sachlichen und finanziellen Ausstattung unbestritten in der Lage, seine satzungsgemäßen Aufgaben wahrzunehmen.

bb) Die streitgegenständliche Werbung der Bekl. verstößt gegen das Werbeverbot für Fernbehandlungen gemäß $\$ 9$ HWG sowohl in dessen alter als auch dessen neuer Fassung und ist damit unlauter i.S.d. $\$ 3$ a UWG.

aaa) Die Bekl. ist Adressatin der Regelung des $₫ 9 \mathrm{HWG}$, sodass sie durch ihre Werbung täterschaftlich gegen diese Vorschrift verstößt. Das Heilmittelwerbegesetz richtet sich an jeden Werbungstreibenden, das heißt an alle natürlichen oder juristischen Personen, die an der Verbreitung einer als Werbung i.S.d. Gesetzes einzustufenden Aussage beteiligt bzw. hierfür verantwortlich sind (OLG München, GRURRR 2020, 461, 462, Rdnr. 38 - digitaler Arztbesuch).

Die Bekl. bewirbt mit den angegriffenen Aussagen gemäß der Anlage K 5 eine Fernbehandlung, nämlich die Erkennung oder Behandlung von Erkältungskrankheiten, die nicht auf eigener Wahrnehmung an dem zu behandelnden Menschen beruht. Es handelt sich hier nicht - wie die Bekl. behauptet - lediglich um eine sachliche Aufklärung. Das Bewerben einer Fernbehandlung durch die Bekl. in der angegriffenen Form ist eine geschäftliche Handlung gemäß sf 3 Abs. 1, 2 Abs. 1 Nr. 1 UWG. Der von $\int S 3$ Abs. 1, 2 Abs. 1 Nr. 1 UWG geforderte objektive Zusammenhang mit der Absatzförderung ist vor allem bei Werbung gegeben (vgl. EuGH, GRUR 2013, 297, Rdnr. 27 - Köck; BGH, GRUR 2010, 161, 163, Rdnr. 11 - Gib mal Zeitung). Die Werbung der Bekl. betrifft die Fernbehandlung durch einen Arzt, vor allem aber auch die eigene Dienstleistung, die als vermittelndes Element dient.

bbb) Die Unlauterkeit der Werbung der Bekl. ergibt sich aus $₫ 3$ a UWG i. V. mit $\$ 9$ HWG.

(1) Die Regelung des $\$ 9 \mathrm{HWG}$ ist dazu bestimmt, im Interesse der Marktteilnehmer das Marktverhalten zu regeln i. S. v. \$3a UWG (KG, GRUR-RS 2019, 40959, Rdnr. 24 - Werbung für ärztliche Fernbehandlung; OLG München, GRUR-RR 2020, 461, 464, Rdnr. 50 - digitaler Arztbesuch, m.w. N.). \$9 S. $1 \mathrm{HWG}$ enthält das Verbot der Werbung für Fernbehandlungen. Der mit Wirkung v. 19.12.2019 eingefügte S. 2 enthält eine Ausnahmevorschrift von diesem Grundsatz. Die Vorschrift dient dem Schutz der Gesundheit der Verbraucher. Diese sollen vor der Gefahr einer unzureichenden Behandlung geschützt werden, die durch die Fernbehandlung, mithin die ausschließliche Verwendung von Mitteln der Fernkommunikation, begründet werden kann. Eine Verletzung dieser Bestimmung ist geeignet, die Interessen der Verbraucher spürbar zu beeinträchtigen (vgl. BGH, GRUR 2012, 647, 651, Rdnr. 42 - INJECTIO; OLG München, GRUR-RR 2020, 461, 464, Rdnr. 50 - digitaler Arztbesuch).

(2) Für den auf Wiederholungsgefahr gestützten Unterlassungsanspruch muss das beanstandete Verhalten der Bekl. sowohl zum Zeitpunkt der Vornahme als auch zum Zeitpunkt der Entscheidung des Senats rechtswidrig sein (st. Rspr; vgl. nur BGH, GRUR 2018, 438, Rdnr. 9 Energieausweis, m.w.N.). \$9 HWG wurde mit Wirkung v. 19.12.1019 - also nach Verkündung des erstinstanzlichen Urteils v. 3.9.2019 - durch den neu angefügten S. 2 ergänzt, der das in S. 1 normierte generelle Werbungsverbot auf die Werbung für bestimmte Fernbehandlungen für nicht anwendbar erklärt. Danach ist hier maßgebend, ob das angegriffene Verhalten zum einen unter Geltung des $\$ 9$ HWG a.F. unzulässig war und zum anderen unter Geltung des $\$ 9 \mathrm{HWG}$ n. F. weiterhin unzulässig ist.
(3) Das angegriffene Verhalten der Bekl. war nach $₫ 9$ HWG a. F. unzulässig und ist dies auch weiterhin unter Geltung des $\$ 9 \mathrm{HWG}$ n. F. Bei dieser gesetzlichen Regelung handelt es sich um ein abstraktes Gefährdungsdelikt (Pfohl, in: Erbs/Kohlhaas, Strafrechtliche Nebengesetze, 232. EL August 2020, \$9 HWG, Rdnr. 1), weshalb es unerheblich ist, ob die Durchführung der Fernbehandlung tatsächlich beabsichtigt oder erfolgt ist (KG, GRUR-RS 2019, 40959, Rdnr. 17 - Werbung für ärztliche Fernbehandlung). Dementsprechend ist auch die einzelne ärztliche Behandlung nicht $\mathrm{zu}$ bewerten, womöglich unter Inanspruchnahme sachverständiger Hilfe. Es geht um die Auslegung der einschlägigen Bestimmungen.

(a) Nach $\$ 9$ HWG a.F. war eine Werbung für die Erkennung oder Behandlung von Krankheiten, Leiden, Körperschäden oder krankhaften Beschwerden unzulässig, die nicht auf eigener Wahrnehmung an dem zu behandelnden Menschen oder Tier beruht (Fernbehandlung). Werbung i. S.d. Heilmittelwerberechts sind alle informationsvermittelnden und meinungsbildenden Aussagen, die darauf abzielen, die Aufmerksamkeit der Adressaten zu wecken und deren Entschlüsse mit dem Ziel der Förderung des Absatzes von Waren oder Leistungen i.S.d. \$1 HWG zu beeinflussen (BGH, NJW 1995, 3054 - Sauerstoff-Mehrschritt-Therapie; Fritzsche, in: Spickhoff, Medizinrecht, 3. Aufl., $\$ 1$ HWG, Rdnr. 5).So liegt es im vorliegenden Fall. Aus der Anlage K 5 lässt sich der werbende Charakter schon anhand der Formulierungen wie „Hier erhalten Sie Ihre AU-Bescheinigung einfach online per Handy nach Hause“ oder „Symptome checken - Risiken ausschließen - Daten eingeben - Einfach bezahlen - Fertig“" ohne weiteres erkennen. Die Aussagen der Bekl. zielen aus Sicht der angesprochenen Verkehrskreise, hier des allgemeinen Verkehrs, zu dem auch die Mitglieder des Senats zählen, konkret darauf ab, Aufmerksamkeit zu wecken und so den Absatz der Dienstleistung der Bekl. zu fördern. Die angegriffene Werbung bewirbt Fernbehandlungen, da der Arzt im konkreten Setting für den angesprochenen Verkehr erkennbar zu keinem Zeitpunkt in unmittelbaren Kontakt mit dem Patienten kommt und die Anamnese auf den Antworten des Patienten auf vorformulierte Fragen beruht.

(b) An der Unzulässigkeit der hier angegriffenen Werbung der Bekl. hat sich auch ab dem 19.12.2019 durch die nach der Verkündung des erstinstanzlichen Urteils ergänzte gesetzliche Regelung des $\$ 9 \mathrm{~S}$. $2 \mathrm{HWG}$ nichts geändert. Nach $\$ 9$ S. 2 HWG ist S. $1-$ an dem sich durch die Neufassung der Vorschrift nichts geändert hat - nicht anzuwenden auf die Werbung für Fernbehandlungen, die unter Verwendung von Kommunikationsmedien erfolgen, wenn nach allgemein anerkannten fachlichen Standards ein persönlicher ärztlicher Kontakt mit dem zu behandelnden Menschen nicht erforderlich ist. \$9 S. 2 HWG wurde in die Vorschrift aufgenommen, um die Anpassung des ärztlichen Berufsrechtes im Hinblick auf die Reichweite des Werbeverbotes nachzuvollziehen (Begr. RegE., BT-Dr. 19/13438, S. 77 f.). Anders als für die Fernbehandlung an sich, kann es im Rahmen der Regelung des $\$ 9$ HWG lediglich auf eine abstrakte, generalisierende Bewertung ankommen, da sich Werbung unabhängig von einer konkreten Behandlungssituation an eine Vielzahl individuell nicht näher individualisierter Personen richtet (Begr. RegE., BT-Dr. 19/13438, S. $77 \mathrm{f}$.). Es dürfen dabei nur solche Fernbehandlungen bei Menschen beworben werden, bei denen die Einhaltung anerkannter fachlicher Standards gesichert ist. Dies ist dann der Fall, wenn nach dem anerkannten medizinischen Stand der Erkenntnisse eine ordnungsgemäße Behandlung und Beratung unter Einsatz von Kommunikationsmedien grundsätzlich möglich ist (Begr. RegE., BT-Dr. 19/13438, S. 77 f.). Dabei ist auch der Gesetzgeber davon ausgegangen, dass der von $\$ 9$ HWG intendierte Schutzbedarf der Patien- 
ten trotz erheblichen Fortschritts der Telemedizin fortbesteht (Begr. RegE., BT-Dr. 19/13438, S. 77).

(aa) Maßstab für die ,,allgemein anerkannten fachlichen Standards" gemäß $\$ 9$ S. 2 HWG ist die MBO-Ä. Unabhängig davon würde sich für die vorliegend relevanten Fragen auch bei Zugrundelegung der BOÄ-SH kein anderer Maßstab ergeben.

Anders als bei der Beurteilung eines konkreten Arztverhaltens im Einzelfall, kommt es für die abstrakte, generalisierende Bewertung des $\$ 9 \mathrm{HWG}$ nicht auf die einzelnen Vorschriften der verschiedenen Landesberufsordnungen an. Auch wenn die MBO-Ä nur Empfehlungscharakter für die Landesärztekammern hat (Scholz, in: Spickhoff, Medizinrecht, 3. Aufl., Vorb. MBO-Ä, Rdnr. 1), stellt sie einen grundlegenden Maßstab für die ärztliche Berufsausübung in Deutschland dar. Sie wird von der Bundesärztekammer erstellt, die eine Arbeitsgemeinschaft der Landesärztekammern ist. Die Beschl. der Bundesärztekammer werden durch einen Ausschuss des Vorstandes, eine Ständige Konferenz der Landesärztekammern und schließlich durch ein zweistufiges Konvergenzverfahren vorbereitet (Scholz, in Spickhoff, Medizinrecht, 3. Aufl., Vorb. MBO-Ä, Rdnr. 1). Die einzelnen Landesärztekammern sind auf diese Weise gemeinsam bei der Erstellung der Vorschriften involviert sodass diese Vorschriften als Konsens mit Blick auf ,allgemein anerkannte fachliche Standards" anzusehen sind. Dies zeigt sich auch daran, dass die einzelnen Berufsordnungen der Länder sich in weiten Teilen an den Vorschriften der $\mathrm{MBO}-\ddot{\mathrm{A}}$ orientieren.

Das Vorbringen der Bekl., der für sie tätige Arzt habe seinen Sitz in S. und die entsprechende landesrechtliche Berufsordnung weiche in dem für den vorliegenden Fall relevanten $\$ 7$ Abs. 4 S. 3 BOÄ-SH vom Wortlaut des $\$ 7$ Abs. 4 S. $3 \mathrm{MBO}-\ddot{\mathrm{A}} \mathrm{ab}$, führt hier nicht zu einem anderen Ergebnis. Zum einen handelt es sich bei $\$ 9 \mathrm{HWG}$ um eine bundesrechtliche Vorschrift, die für die Werbung für Fernbehandlungen, die naturgemäß überregional erfolgen kann, auf ,allgemein anerkannte fachliche Standards“ abstellt. Diese ,allgemein anerkannten Standards“ hängen nicht unmittelbar davon $a b$, ob eine einzelne Landesvorschrift womöglich abweichend gefasst ist. Sie sind vielmehr Ausdruck überregional übereinstimmender Regeln, die von den einzelnen Landesvorschriften $\mathrm{zu}$ trennen sind. Zum anderen betrifft $\$ 9 \mathrm{HWG}$ lediglich die zulässige Bewerbung einer Fernbehandlung und nicht die Zulässigkeit einer Fernbehandlung an sich. Der einzelne Arzt ist durch dieses Ergebnis nicht in der Behandlung von Patienten beschränkt, sondern nur in der Bewerbung spezieller Behandlungsmethoden. Der Maßstab der MBO-Â als konsensualer Standard für das Bundesgebiet ermöglicht eine einheitliche und interessengerechte Auslegung des $\$ 9 \mathrm{HWG}$.

Im Übrigen ergibt sich aus $\$ 7$ Abs. 4 S. 3 BOÄ-SH für das im vorliegenden Fall maßgebende Merkmal des „Einzelfalls" kein anderer Maßstab als aus $\$ 7$ Abs. 4 S. 3 MBO-Ä. Nach $\$ 7$ Abs. 4 S. 3 MBO- $\ddot{A}$ ist eine ausschließliche Beratung oder Behandlung über Kommunikationsmedien im Einzelfall erlaubt, wenn dies ärztlich vertretbar ist und die erforderliche ärztliche Sorgfalt insbesondere durch die Art und Weise der Befunderhebung, Beratung, Behandlung sowie Dokumentation gewahrt wird und die Patientin oder der Patient auch über die Besonderheiten der ausschließlichen Beratung und Behandlung über Kommunikationsmedien aufgeklärt wird. Demgegenüber enthält $\$ 7$ Abs. 4 S. 3 BOÄ-SH keine ausdrückliche Bezugnahme auf den Einzelfall. Danach ist eine Beratung oder Behandlung vorbehaltlich anderweitiger gesetzlicher Regelungen ausschließlich über Kommunikationsmedien erlaubt, wenn diese ärztlich vertretbar und ein persönlicher Kontakt mit der Patientin oder dem Patienten nicht erforderlich ist. Dass dieser Unterschied im Wortlaut jedoch - anders als die Bekl. geltend macht - keinen niedrigeren Prüfungs- maßstab im Rahmen des $₫ 7$ Abs. 4 S. 3 BOÄ-SH begründet, ergibt sich insbesondere aus $\$ 2 \mathrm{BOA}-\mathrm{SH}$, der die allgemeinen ärztlichen Berufspflichten vorgibt. Der Arzt soll u.a. seinen Beruf nach seinem Gewissen, den Geboten der ärztlichen Ethik und der Menschlichkeit ausüben (Abs. 1), sein ärztliches Handeln am Wohl der Patientinnen und $\mathrm{Pa}-$ tienten ausrichten und seinen Beruf gewissenhaft ausüben (Abs. 2), wobei eine gewissenhafte Ausübung des Berufs insbesondere die notwendige fachliche Qualifikation und die Beachtung des anerkannten Standes der medizinischen Erkenntnisse erfordert (Abs. 3). Diese grundlegenden Berufspflichten sind einzelne Konkretisierungen der umfassend geltenden ärztlichen Sorgfalt, welche den einzelnen Vorschriften jeder BOÄ immanent ist. Die Frage, ob eine Fernbehandlung ,ärztlich vertretbar“ ist, hängt also unmittelbar mit dem Wohl der Patientinnen und Patienten zusammen. $\mathrm{Ob}$ ein persönlicher Kontakt für das Wohl des einzelnen Patienten notwendig ist, kann denklogisch nur im konkreten Arzt-Patienten-Verhältnis beurteilt werden. Die Einhaltung ärztlicher Sorgfalt setzt also voraus, dass der Arzt ,im konkreten Einzelfall“" prüft, ob eine Behandlung ausschließlich über Kommunikationsmedien ärztlich vertretbar ist. Die ärztliche Vertretbarkeit kann dabei nicht losgelöst vom Einzelfall beurteilt werden, sodass eine Einzelfallprüfung über das Merkmal der ärztlichen Vertretbarkeit auch im Rahmen des $₫ 7$ Abs. 4 S. 3 BOÄ-SH notwendig ist.

(bb) Die Werbung der Bekl. erfüllt die Anforderungen des \$9 S. 2 HWG für eine ausnahmsweise Zulässigkeit nicht. Das streitgegenständliche Setting berücksichtigt nicht die notwendige Einzelfallprüfung des $\$ 7$ Abs. 4 MBO-Ä und entspricht insoweit nicht den ,allgemein anerkannten fachlichen Standards“ i.S.d. \$9 S. 2 HWG. Das grundsätzliche Verbot der Werbung für Fernbehandlungen dient vorrangig dem Schutz der Volksgesundheit und des individuellen Gesundheitsinteresses und basiert auf dem Grundgedanken, dass partielle Informationen, seien diese auch wissenschaftlich objektivierbar, nie das gesamtheitliche Bild ersetzen können, das sich der Heilkundige bei persönlicher Wahrnehmung und Untersuchung des Patienten machen kann. Die Werbung für derartig verkürzte Behandlungsmethoden soll grundsätzlich unterbunden werden, unabhängig davon, ob die Fernbehandlung im Einzelfall erlaubt ist (vgl. KG, GRUR-RS 2019, 40959, Rdnr. 17 - Werbung für ärztliche Fernbehandlung).

Nach $\$ 7$ Abs. 4 S. 1 MBO-Ä sollen Ärzte Patientinnen und Patienten im persönlichen Kontakt beraten und behandeln. Gemäß S. 3 ist, wie ausgeführt, eine ausschließliche Beratung oder Behandlung über Kommunikationsmedien im Einzelfall erlaubt, wenn dies ärztlich vertretbar ist und die erforderliche ärztliche Sorgfalt insbesondere durch die Art und Weise der Befunderhebung, Beratung, Behandlung sowie Dokumentation gewahrt wird und die Patientin oder der Patient auch über die Besonderheiten der ausschließlichen Beratung und Behandlung über Kommunikationsmedien aufgeklärt wird. Die Regelung in $\$ 7$ Abs. 4 S. 3 MBO-Ä legt den Ärztinnen und Ärzten die Pflicht auf, im konkreten Einzelfall zu entscheiden, ob eine Fernbehandlung mit dem anerkannten Stand der medizinischen Erkenntnisse vereinbar ist (Begr. RegE., BTDr. 19/13438, S. 77 f.). Die Bundesärztekammer hat in der Begründung zur Neufassung des $\$ 7 \mathrm{MBO}-\ddot{A}$ ausdrücklich festgehalten, „dass der Grundsatz der ärztlichen Beratung und Behandlung im persönlichen Kontakt zwischen Arzt und Patient, das heißt unter physischer Präsenz der Ärztin oder des Arztes, zu erfolgen hat und weiterhin den ,Goldstandard 'ärztlichen Handelns in Beziehung zu den Patientinnen und Patienten darstellt." Für die Beratung und Behandlung ausschließlich aus der Ferne ist im Einzelfall ,zu prüfen, ob die erforderliche ärztliche Sorgfalt insbesondere durch die Art und Weise der Befunderhebung, die Bera- 
tung, die Behandlung sowie die Dokumentation gewahrt wird" (Beschlussprotokoll des 121. Deutschen Ärztetags, TOP IV - 01, S. 2f.).

Der „Einzelfall“ i.S.d. $\$ 7$ Abs. 4 S. 3 MBO-Ä meint das konkrete Arzt-Patienten-Verhältnis und nicht - wie die Bekl. meint - ein spezielles Krankheitsbild, wie z.B. das einer Erkältung. Die Neufassung der Vorschrift soll die Fort- und Weiterentwicklung telemedizinischer, digitaler, diagnostischer und anderer vergleichbarer Möglichkeiten berücksichtigen, aber weiterhin den Grundsatz der Behandlung im persönlichen Kontakt verdeutlichen (Beschlussprotokoll des 121. Deutschen Ärztetags, TOP IV - 01, S. 2f.). Es ist das grundlegende Ziel, dass konkrete und individuell auf den einzelnen Patienten abgestellte Diagnosen gestellt oder Therapieempfehlungen gegeben werden. Dies muss auch berücksichtigt werden, wenn es um die Frage geht, in welchen Fällen eine Fernbehandlung möglich sein soll.

Entgegen der Ansicht der Bekl. ergibt sich dieses Ergebnis auch aus den „Hinweisen und Erläuterungen zu $\$ 7$ Abs. 4 MBOErläuterung zum Tatbestandsmerkmal des „Einzelfalls“ ausgeführt, dass ,die Ärztin bzw. der Arzt jeweils in Bezug auf den einzelnen Behandlungs- bzw. Beratungsfall unter Berücksichtigung sämtlicher Begleitumstände zu prüfen [hat], ob dieser für eine ausschließliche Fernbehandlung im Sinne der Vorschrift geeignet ist" (,Hinweise und Erläuterungen zu $\$ 7$ Abs. 4 MBO-Ä“", Stand: 22.3.2019, II.3., Anlage K 11). Schon nach dem Wortlaut der Erläuterungen kann sich der einzelne Beratungs- und Behandlungsfall nur auf das konkrete Arzt-Patienten-Verhältnis beziehen. $\mathrm{Zu}$ den Begleitumständen zählt darüber hinaus ausdrücklich der Bezug zu der Patientin oder dem Patienten (III.4. der Hinweise und Erläuterungen). Der „Einzelfall“ i.S.d. \$7 Abs. 4 S. 3 MBO- Ä kann sich nach diesen Ausführungen nicht unabhängig vom einzelnen Patienten lediglich auf ein Krankheitsbild beziehen, sondern meint gerade ein konkretes Arzt-Patienten-Verhältnis.

(cc) Unter Berücksichtigung dieser Grundsätze erfüllt die Werbung der Bekl. nicht die Voraussetzungen des $\$ 9$ S. 2 HWG. Im Rahmen des Geschäftsmodells der Bekl. muss der Interessent zunächst online verschiedene Fragen beantworten, die vor allem die Symptome und die Risikofaktoren des Nutzers betreffen. Wenn die Antworten zu der Diagnose einer Erkältung passen, werden sie an den „Tele-Arzt“ gesendet, welcher die mitgeteilten Symptome überprüft und eine entsprechende AU-Bescheinigung ausstellt, sollten die mitgeteilten Symptome mit der vermuteten Diagnose einer Erkältung übereinstimmen. Wenn die Antworten auf die voreingestellten Fragen nicht zu der entsprechenden Diagnose führen, erscheint eine Meldung, wonach der Patient den Dienst nicht nutzen könne. Es steht dem Patienten dann allerdings offen, die Fragen erneut zu beantworten. Wenn der Arzt eine AU-Bescheinigung ausstellt, wird diese per WhatsApp und per Post an den Nutzer geschickt. Der Arzt kommt zu keinem Zeitpunkt in Kontakt mit dem Patienten, es sei denn er hält Rückfragen per Telefon oder Video-Chat für notwendig. Zu diesem Fall kommt es im streitgegenständlichen Setting aber voraussehbar nicht, da dem Arzt nur solche Antworten übermittelt werden, die zur Diagnose einer Erkältung passen. Fälle, in denen Rückfragen angezeigt wären, werden vom System vorzeitig aussortiert. Die Anamnese beruht in diesem Setting ausschließlich auf den Antworten des Patienten auf die vorformulierten Fragen. Eine Abwägung im Einzelfall kann auf diese Weise nicht stattfinden. Das würde vielmehr voraussetzen, dass sich der behandelnde Arzt einen umfassenden Eindruck vom Gesundheitszustand des Patienten verschafft. Dies ist durch das voreingestellte Setting im Rahmen des beworbenen Geschäftsmodells gerade nicht möglich.
Anders als die Bekl. meint, führt dieses Ergebnis auch nicht zu einer generellen Unzulässigkeit telemedizinischer Behandlungen, was dem Willen des Gesetzgebers widerspräche. Dies liegt zunächst schon daran, dass $\$ 9 \mathrm{HWG}$ nur die Bewerbung der Fernbehandlung und nicht die Zulässigkeit der Fernbehandlung als solche betrifft. Darüber hinaus schließt dieses Ergebnis auch Fernbehandlungen als solche nicht aus, da lediglich eine Prüfung des konkreten Einzelfalls hinsichtlich der medizinischen Vertretbarkeit einer ausschließlichen Fernbehandlung vorgenommen werden muss. Eine telemedizinische Behandlung kann erhebliche Vorteile bieten, die dem Patienten nicht vorenthalten werden sollen. Dies gilt aber nur, soweit der behandelnde Arzt überprüfen kann, ob ein persönlicher Kontakt zum Patienten im konkreten Einzelfall tatsächlich nicht notwendig ist, wie z. B. im Rahmen einer Videosprechstunde.

Auch der Einwand der Bekl., ihr seien nach über 70.000 Ferndiagnosen keinerlei Fehldiagnosen gemeldet worden, vermag an diesem Ergebnis nichts zu ändern. Unabhängig davon, ob bisher tatsächlich keine Fehldiagnosen gestellt wurden oder diese aus anderen Gründen nicht an die Bekl. herangetragen wurden, führt dieser Einwand nicht zu einem Wegfall der notwendigen Prüfung im konkreten Einzelfall.

(dd) Dieses Ergebnis steht auch im Einklang mit den neuesten Entwicklungen während der derzeitigen COVID19-Pandemie. Während der Pandemie besteht ein erhebliches Interesse daran, dass Patienten mit entsprechenden Symptomen eine AU-Bescheinigung erhalten, ohne durch einen physischen Besuch beim Arzt das Ansteckungsrisiko vor Ort zu vergrößern. $\int 8$ Abs. 1 der einschlägigen $A U-$ RL sieht insoweit vor, dass der G-BA eine Ausnahme der grundsätzlichen Regel des $\$ 4$ AU-RL durch Beschl. insofern zulassen kann, dass die Feststellung der Arbeitsunfähigkeit bei Versicherten mit Erkrankungen der oberen Atemwege, die keine schwere Symptomatik vorweisen, für einen Zeitraum von bis zu 7 Kalendertagen auch nach telefonischer Anamnese und zwar im Wege der persönlichen ärztlichen Überzeugung vom Zustand der oder des Versicherten durch eingehende telefonische Befragung erfolgen darf. Das Fortdauern der Arbeitsunfähigkeit kann danach im Wege der telefonischen Anamnese einmalig für einen weiteren Zeitraum von bis zu 7 Kalendertagen festgestellt werden. Von diesen Möglichkeiten hat der G-BA im Frühjahr 2020 befristet Gebrauch gemacht.

Die Möglichkeit der telefonischen Anamnese ist eine Maßnahme der Risikominimierung in einer Ausnahmesituation während der COVID-19-Pandemie. Daran lässt sich deutlich erkennen, dass eine Fernbehandlung in Form der Bescheinigung der Arbeitsunfähigkeit, wie im Rahmen der mündlichen Verhandlung vor dem Senat angesprochen, nicht einmal in dieser Ausnahmesituation mit geringerem persönlichem Kontakt als einem Telefonat zulässig sein soll. Dies kann dann erst recht nicht außerhalb dieses Ausnahmezustands zulässig sein. Innerhalb des Settings der Bekl. kommt es voraussehbar zu keinem Zeitpunkt zu einem (telefonischen) Kontakt zwischen Arzt und Patient. Der Arzt erhält lediglich die Antworten auf vorformulierte Fragen übermittelt, die die Annahme einer Erkältung nahelegen. In dieser Situation hat der Arzt typischerweise keinen Anlass für telefonische Rückfragen. Dass die Fragen unter Umständen bereits mehrfach beantwortet wurden, bevor eine Übermittlung erfolgte, kann er nicht erkennen.

cc. Es besteht schließlich auch Wiederholungsgefahr. Eine solche liegt vor, wenn eine Wiederholung des wettbewerbswidrigen Verhaltens ernsthaft und greifbar zu besorgen, nicht schon, wenn sie nur denkbar oder möglich ist (Bornkamm, in: Köhler/Bornkamm/Feddersen, 38. Aufl., \8 UWG, Rdnr. 1.42). Ein erfolgter Wettbewerbsverstoß begründet eine tatsächliche Vermutung für die Wiederho- 
lungsgefahr (st. Rspr., vgl. nur BGH, GRUR 2020, 755, 763, Rdnr. 80 - WarnWetter-App, m. w. N.). Die Widerlegung dieser Vermutung kann durch die Abgabe einer strafbewehrten Unterlassungsverpflichtungserklärung erreicht werden (vgl. z. B. BGH, GRUR 2020, 755, 763, Rdnr. 81 WarnWetter-App). Eine solche Unterlassungserklärung hat die Bekl. indes nicht abgegeben. Andere Anhaltspunkte, die die tatsächliche Vermutung widerlegen könnten, sind weder vorgetragen noch sonst ersichtlich.

b. Die Abmahnkosten sind gemäß $\$ 12$ Abs. 1 S. 2 UWG in der geltend gemachten Höhe zu erstatten. Die Abmahnung war begründet und berechtigt, wobei es auf die Rechtslage zum Zeitpunkt der Abmahnung (\$9 HWG a. F.) ankommt (st. Rspr.; vgl. nur BGH, GRUR 2018, 438, Rdnr. 9 - Energieausweis, m. w. N.). Auch der Höhe nach besteht der geltend gemachte Anspruch. Da die Bekl. insoweit keine spezifizierten Einwendungen erhoben hat, sind weitere Ausführungen des Senats entbehrlich.

$[\ldots]$

4. Die Voraussetzungen für eine Zulassung der Revision nach $\$ 543$ Abs. 2 ZPO liegen nicht vor. Die Rechtssache hat weder grundsätzliche Bedeutung noch erfordern die Fortbildung des Rechts oder die Sicherung einer einheitlichen Rechtsprechung eine Entscheidung des Revisionsgerichts. Auch wenn $\$ 9$ S. 2 HWG vor kurzem geändert worden ist, ändert dies nichts daran, dass es sich vorliegend um eine Einzelfallentscheidung hinsichtlich der konkret angegriffenen Werbung handelt. Die getroffene Entscheidung steht im Einklang mit der bekannten ober- und höchstrichterlichen Rechtsprechung. Etwas Abweichendes trägt auch die Bekl. nicht vor.

\section{Anmerkung zu OLG Hamburg, Urt. v. 5.11.2020 - 5 U 175/19 (LG Hamburg) \\ Corinna Schmidt-Murra}

Die Entscheidung des OLG Hamburg ist dem Grunde nach begrüßenswert, weil sie anders als das OLG München $^{1}$ vor einer Sachverhaltssubsumtion erstmals den ausführlich begründeten Versuch unternimmt, den für die Zulässigkeit der Werbung für eine Fernbehandlung gem. der Ausnahmevorschrift des \$9 S. 2 HWG n. F. zu beachtenden Maßstab der allgemein anerkannten fachlichen Standards abstrakt zu definieren. Allerdings erweist sich die Bezugnahme auf die MBO- $\ddot{A}$ weder als konsistent mit der Gesetzesbegründung, noch wird der Maßstab darin hinreichend definiert. Bedauerlich ist zudem, dass das Gericht die bei der Ausstellung ärztlicher Atteste zu beachtenden Bedingungen hiervon nicht abgrenzt und weiter vertieft.

\section{1. $\$ 7$ Abs. 4 S. 3 MBO-Ä als Maßstab für die ,allgemein anerkannten fachlichen Standards" gemäß \$9 S. 2 HWG?}

\9 S. 2 HWG erlaubt die Werbung für eine Fernbehandlung, wenn die ,allgemein anerkannten fachlichen Standards“ einen persönlichen ärztlichen Kontakt mit dem zu behandelnden Menschen nicht erfordern. Diese materiellen Standards werden durch das HWG allerdings nicht unmittelbar selbst definiert, weshalb das OLG Hamburg insofern die $\mathrm{MBO}-\ddot{\mathrm{A}}$ bemüht.

Rechtsanwältin Dr. iur. Corinna Schmidt-Murra, SCHMIDT, VON DER OSTEN \& HUBER Rechtsanwälte Steuerberater Partnerschaft mbB, Rüttenscheider Str. 26, 45128 Essen, Deutschland

\section{a) Fehlende Rechtsqualität der $M B O-\dot{A}$}

Das Gericht stellt sich damit allerdings gegen die gefestigte Auffassung, dass die $\mathrm{MBO}-\ddot{A}$ aufgrund ihres bloßen Empfehlungscharakters rechtlich nicht verbindlich ist, weshalb für die Beurteilung der Rechtmäßigkeit ärztlichen Verhaltens stets die jeweiligen Berufsordnungen der Kammern heranzuziehen sind ${ }^{2}$. Auch wenn es wegen der häufigen Wortlautidentität der $\mathrm{MBO}-\ddot{\mathrm{A}}$ mit der jeweiligen Berufsordnung auf diese Unterscheidung zumeist nicht ankommt ${ }^{3}$, wurde dieses Verhältnis vorliegend tatsächlich relevant. Denn anders als $₫ 7$ Abs. 4 S. 3 MBO-̈̈ enthält $₫ 7$ Abs. 4 S. 3 BO- Ä Schleswig-Holstein nicht die von dem Gericht als relevant erachtete „Einschränkung“, dass nur ,im Einzelfall“ eine Beratung oder Behandlung ausschließlich über Kommunikationsmedien erlaubt ist, wenn diese ärztlich vertretbar und ein persönlicher Kontakt mit der Patientin oder dem Patienten nicht erforderlich ist.

Sofern sich daraus regionale Unterschiede für die $\mathrm{Zu}-$ lässigkeit einer Fernbehandlungsmaßnahme ergeben, wäre dies ein hinzunehmender Ausfluss der durch die Heilberufsgesetze allein den Landesärztekammern zugewiesenen Kompetenz ${ }^{4}$. Ferner ist zu bedenken, dass dem Arzt ohnehin - unabhängig von $\$ 9 \mathrm{HWG}$ - eine Werbung für eine berufsrechtswidrige Fernbehandlung bereits durch die berufsrechtlichen Vorschriften, namentlich $\$ 27$ Abs. 3 S. 1 MBO- $\ddot{A}$ in Verbindung mit $\int 7$ Abs. 4 S. $3 \mathrm{MBO}-\ddot{A}$ in der Fassung der jeweiligen Berufsordnungen der Landesärztekammern, untersagt wird und hierbei regionale Unterschiede selbstverständlich - was auch der Gesetzgeber letztlich selbst unterstellt ${ }^{5}-$ beachtlich wären.

\section{b) Entgegenstehende Gesetzesbegründung}

Ungeachtet dessen ist der Verweis auf die MBO- $\ddot{A}$ auch deshalb kritikwürdig, weil die Gesetzesbegründung zu der Novellierung des $\$ 9 \mathrm{HWG}$ - die das Gericht auch selbst referenziert - diese Schlussfolgerung nicht trägt.

Nach Auffassung des Gesetzgebers konnte das Verbot des \$9 HWG nicht einfach gänzlich gestrichen werden, weil auch Situationen denkbar seien, bei denen der Werbende nicht den Vorgaben einer rechtlich verbindlichen Berufsordnung unterworfen sei und somit ein Schutzbedarf fortbestehe $^{6}$. Bereits ausgehend von diesem Ansatz muss die Zulässigkeit der Werbung für eine Fernbehandlung nach dem Willen des Gesetzgebers folglich losgelöst von den berufsechtlichen Bestimmungen erfolgen. Hinzukommt die gesetzgeberische Feststellung der unterschiedlichen Umsetzung der Anpassung des $\$ 7$ Abs. 4 der MBO- $\ddot{A}$ in den einzelnen Berufsordnungen auf Länderebene.

Der Gesetzgeber gelangt schließlich zu der konsequenten Bewertung, dass es ,im Gegensatz zu der berufsrechtlich vorgesehenen, konkreten und individuellen Einzelfallentscheidung [...] im Rahmen der Regelung des $\$ 9$ HWG lediglich auf eine abstrakte, generalisierende Bewertung ankommen“ könne, ,da sich Werbung unabhängig von einer konkreten Behandlungssituation an eine Vielzahl individuell nicht näher individualisierter Personen" richte $^{7}$. Der Gesetzgeber entscheidet sich damit ausdrücklich gegen eine unmittelbare Bezugnahme auf die berufs-

1) MedR 2021, 154 m. Anm. Braun.

2) BGH, GRUR 2000, 1080, 1082; zu \$7 Abs. 4 S. 3 MBO-Ä: OLG München, BeckRS 2016, 12692, Rdnr. 39.

3) BGH, GRUR 2000, 1080, 1082; bei $\$ 7$ Abs. 4 S. 3 MBO-Ä eine Wortlautidentität unterstellend OLG München, BeckRS 2016, 12692, Rdnr. 39.

4) Vgl. Scholz, in: Spickhoff, Medizinrecht, 3. Aufl. 2018, MBO-Ä, Vorb., Rdnr. 1, MWBO, Vorb., Rdnr. 6.

5) BT-Dr. 19/13438, S. 78.

6) BT-Dr. $19 / 13438$, S. 77

7) BT-Dr. 19/13438, S. 78. 